\title{
СИНТЕЗ РАДИАЛЬНО-БАЗИСНЫХ НЕЙРОННЫХ СЕТЕЙ С ПРЕОБРАЗОВАНИЕМ НА ОБОБЩЕННУЮ ОСЬ ДЛЯ АВТОМАТИЗАЦИИ ПРОЦЕССА ПРИНЯТИЯ ДИАГНОСТИЧЕСКИХ РЕШЕНИЙ
}

\author{
Субботин С.A. ${ }^{1}$ (Subbotin S.A.) \\ 13апорожский национальный технический университет, Запорожье \\ E-mail: subbotin@zntu.edu.ua \\ ORCID: 0000-0001-5814-8268
}

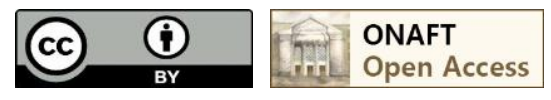

DOI: $10.15673 /$

\begin{abstract}
Аннотация
Решена проблема синтеза радиально-базисных нейронных сетей на основе набора прецедентов для автоматизации принятия решений в диагностике. Предложен метод синтеза радиально-базисных нейронных сетей, который использует отображение выборки из многомерного пространства признаков на одномерную обобщенную ось для выделения центров и границ кластеров и, в отличие от известных методов, не требует задания пользователем числа кластеров, не имеет неопеделенности выбора числа нейронов в первом слое и выбора начальных значений весов сети, стремится минимизировать размер сети, характеризуется приемлемым временем обучения, учитывает информативность признаков при формировании разбиения на кластеры, благодаря использованию процедуры оптимизации сети позволяет получать безызбыточные контрастные интерпретабельные модели, а также обеспечивает возможность дообучения ранее построенных моделей. Разработано программное обеспечение, реализующее предложенный метод, а также проведены эксперименты, подтвердившие работоспособность разработанного математического обеспечения и позволяющие рекомендовать его для использования на практике. Установлено, что предложенный метод характеризуется менышими затратами времени на построение радиально-базисных нейронных сетей по сравнению с использованием четкого переборного кластерного анализа и большими затратами времени по сравнению с отображением выборки в кластеры. При этом предложенный метод обеспечивает приемлемую точность и существенно более высокий уровень обобщения по сравнению с известными методами.
\end{abstract}

\section{Abstract}

The problem of synthesis of radial basis neural networks based on a set of precedents for the automation of decision-making in the diagnosis is solved. The method of radial basis neural network synthesis is proposed. It uses a transformation mapping a sample from the multidimensional feature space into one-dimensional generalized axis to allocate cluster centers and boundaries and, in contrast to the known methods, does not require the user's involvement in clusters number selection, has no uncertainty in the selection of the number of neurons in the first layer and the in the choice of the initial weight values of the network, aims to minimize the size of the network, characterized by an acceptable training time, at the clusters partition formation takes into account the feature informativities, through the use of network optimization procedure allows to obtain non-redundant interpretable contrast models, and allows additional training of previously built models. The software that implements the proposed method is developed. Experiments were conducted to study the method. They confirmed its efficiency and allow to recommend the method to be used in practice. It is found that the proposed method is characterized by a less time-consuming to build a radial basis neural-networks compared to using clear exhaustive search of the cluster 


\section{ТЕОРІЯ І ПРАКТИКА АВТОМАТИЗАЩЇ БІЗНЕС-ПРОЦЕСІВ}

analysis and time-consuming in comparison with the mapping samples in clusters. Thus the proposed method provides acceptable accuracy and significantly higher level of abstraction than the known methods. Key words: neural network, radial base network, training, synthesis, diagnostics.

\section{Ключевые слова}

Нейронная сеть, радиально-базисная сеть, обучение, синтез, диагностика.

Введение. Задачи автоматизации принятия решений получили широкое распространение в различных областях науки и техники, в частности, в технической и биомедицинской диагностике. Особо можно выделить ситуацию, когда отсутствует аналитическая модель зависимости состояния диагностируемого объекта, экспертное знание об этой зависимости отсутствует или является неполным, а сама зависимость полагается многомерной и нелинейной. В этом случае задача автоматизации принятия решений, как правило, решается путем построения диагностической модели по прецедентам - экспериментально полученным наблюдениям, точечно характеризующим моделируемую зависимость [1,2].

В качестве базиса для построения моделей по прецедентам, как правило, используют искусственные нейронные сети [3-11] - однородные вычислительные структуры, обладающие способностями обучаться по примерам: обобщать данные и неявно извлекать знания из данных, отображая их в структуру и параметры (веса) сети.

Известно достаточно много различных моделей нейронных сетей и методов их обучения [3-11]. Однако для задач автоматизации принятия решений целесообразно использовать преимущественно НС прямого распространения сигнала, которые не содержат боковых и обратных связей и легче поддаются последующему анализу $[6,9,10]$. Среди НС данного класса особо можно выделить радиально-базисные НС (РБНС), которые состоят из двух слоев нейронов и легко интерпретируются в терминологии кластерного анализа, что упрощает последующий анализ полученной на их основе модели или её решений $[3,6,11,12]$.

Известные методы построения РБНС можно разделить на две группы.

Методы первой группы предполагают, что число нейронов РБНС в первом слое задается пользователем, а число нейронов во втором слое определяется размерностью выхода сети. После чего РБНС рассматривается как частный случай многослойной нейронной сети прямого распространения сигнала (многослойного персептрона), которую обучают с помощью градиентных методов многомерной нелинейной безусловной оптимизации [8, 12], а частные производные целевой функции определяют на основе техники обратного распространения ошибки [8, 10 , 11]. При этом существует неопределенность выбора числа нейронов в первом слое, что может привести к получению избыточной сети или, наоборот, к невозможности построения модели, обладающей требуемой точностью. Другим недостатком данных методов является неопределенность выбора начальных значений весов сети, что может привести к невозможности решить задачу обучения РБНС за ограниченное время.

Методы второй группы предполагают, что перед построением РБНС решена задача кластер-анализа [12] и выделены центры кластеров. Число нейронов в первом слое РБНС задают равным числу кластеров, а в веса нейронов первого слоя заносят координаты центров кластеров. Далее РБНС обучают, корректируя веса нейронов второго слоя на основе методов многомерной нелинейной безусловной оптимизации [8, 12]. При этом качество полученной нейромодели существенно зависит от качества результатов кластер-анализа, а время обучения существенным образом определяется временем кластер-анализа.

Целью данной работы являлось создание метода структурно-параметрического синтеза РБНС, свободного от перечисленных недостатков.

\section{Постановка задачи}

Пусть задана обучающая выборка прецедентов $<x, y>$, где $x=\left\{x^{s}\right\}, s=1,2, \ldots, S, S$ - число прецедентов $\left\{x^{s}\right\}$, $x=\left\{x_{j}\right\}, x^{s}=\left\{x_{j}^{s}\right\}, j=1,2, \ldots, N, x_{j}^{s}$-значение $j$-го диагностического признака $x_{j}$, характеризующее прецедент (экземпляр) $x^{s}, N$ - число диагностических признаков, $y=\left\{y^{s}\right\}, y^{s}$-значение выходного признака, сопоставленное прецеденту $x^{s}$.

Тогда задача структурно-параметрического синтеза РБНС заключается в том, чтобы получить $\langle f(), w>$ : $y^{s^{*}}=F\left(w, x^{s}\right), f(F(), w,<x, y>) \rightarrow o p t$, где $f()$ - пользовательский критерий, характеризующий качество аргумента относительно решаемой задачи, opt-оптимальное (желаемое или приемлемое) значение функционала $f()$ для решаемой задачи, $F()$ - структура РБНС, $w$ - множество управляемых (настраиваемых) параметров (весов) РБНС, 


\section{ТЕОРІЯ І ПРАКТИКА АВТОМАТИЗАЦІЇ БІЗНЕС-ПРОЦЕСІВ}

$\left\langle F(), w>\right.$ - диагностическая модель на основе РБНС, которая задаётся кортежем $\left\langle M,\left\{N_{\eta}\right\},\left\{y^{(\eta, i)}\left(x^{(\eta, i)}\right)\right\}>\right.$ и описывается функционально формулами:

$$
\begin{gathered}
y_{i}^{s}=y^{(M, i)}\left(y^{(M-1, i)}\left(\ldots . y^{(1, i)}\left(x^{s}\right)\right)\right), i=1,2, \ldots, N_{\eta-1}, w^{(\eta, i)}=\left\{w_{j}^{(\eta, i)}\right\}, \\
y^{(0, j)}=\psi^{(0, j)}=x_{j}^{s}, N_{0}=N, x_{j}^{(1, i)}=x_{j}^{s}, i=1,2, \ldots, N_{\eta-1}, \eta=1,2, \ldots, M, j=1,2, \ldots, N .
\end{gathered}
$$

Здесь $\left\{y^{(\eta, i)}=\psi^{(\eta, i)}\left(\varphi^{(\eta, i)}\right)\right\}$ и $w=\left\{w^{(\eta, i)}\right\}=\left\{w_{j}^{(\eta, i)}\right\}-$ соответственно, структурные блоки и параметры РБНС, $M-$ число слоёв РБНС $(M=2), N_{\eta}$ - число узлов в $\eta$-м слое, $y^{s}=\left\{y_{i}^{s}\right\}, i=1,2, \ldots, N_{M}, y_{i}^{s}-$ значение $i$-го выходного признака для экземпляра $x^{s} ; N_{M}$ - число выходных признаков, $\varphi^{(\eta, i)}, \psi^{(\eta, i)}-$ соответственно, дискриминантная (весовая) и активационная функции $i$-го узла $\eta$-го слоя, $y^{s^{*}}=\psi^{(M, i)}\left(x^{s}\right)$ - расчётное значение на $i$-м выходе РБНС для экземпляра $x^{s}$, поданного на её входы, $w_{j}^{(\eta, i)}$ - значение $j$-го настраиваемого параметра или веса $j$-го входа $i$-го узла $\eta$ го слоя. Для задач классификации: $y^{s} \in\{q\}, q=1,2, \ldots, K, K$ - число классов $K, K>1$;

В простейшем случае $f$ определяют: $f=E$, где $E$ - среднеквадратическая ошибка:

$$
E=\frac{1}{2} \sum_{s=1}^{S}\left(y^{s}-\psi^{(M, i)}\left(x^{s}\right)\right)^{2} \rightarrow \min .
$$

Метод синтеза радиально-базисных нейронных сетей с отображением на обобщённую ось

Из рассмотренных выше двух основных групп методов обучения РБНС методы на основе кластер-анализа обладают меньшей неопределенностью. Поэтому их использование представляется более целесообразным. Однако данные методы существенно зависят от кластер-анализа: при большом числе признаков, характеризующих экземпляры, потребуется расчет расстояний в многомерном пространстве признаков между всеми экземплярами, что повлечет за собой существенные затраты времени, а также может в ряде случаев приводить к получению плохого разбиения пространства признаков на кластеры вследствие подавления большим числом малоинформативных признаков малого числа высокоинформативных признаков.

Для устранения данных недостатков представляется целесообразным использовать кластер-анализ в одномерном пространстве обобщенного признака, на ось которого отображать экземпляры из исходного пространства признаков. При этом расстояния между экземплярами будут определяться в одномерном пространстве, а также исключат подавление информативных признаков малоинформативными в процессе выделения кластеров.

В качестве преобразования на обобщённую ось целесообразно использовать процедуру, не требующую расчета расстояний между экземплярами и позволяющую учесть информативность признаков, сохраняя топологию классов.

После получения кластеров на одномерной оси для их экземпляров возможно получить координаты центров в исходном пространстве признаков, которые занести в веса нейронов первого слоя РБНС, а нейроны второго слоя предлагается обучать стандартным способом - на основе обратного распространения ошибки [7, 10, 11] или решения системы линейных алгебраических уравнений [9].

Метод синтеза РБНС, реализующий приведенные выше идеи, предполагает выполнение следующих этапов.

Этап 1. Инициализация. Задать допустимый предел ошибки $\varepsilon$, а также обучающую выборку прецедентов $<x$, $y>$. Определить $x_{j}^{\min }, x_{j}^{\max }-$ минимальное и максимальное значения $j$-го признака, $j=1,2, \ldots, N$, a также $y_{i, \max }, y_{i, \min }-$ максимальные и минимальные значения выходных признаков, $i=1,2, \ldots, N_{M}$.

При необходимости (для задач оценивания) дискретизировать значения выходного признака, заменив их номерами псевдоклассов. Дискретизацию можно выполнить, используя формулу:

$$
y_{i}^{s}=\left(y_{i}^{s}-y_{i, \text { min }}\right) \operatorname{div}\left(\frac{y_{i, \max }-y_{i, \min }}{K}\right),
$$

где $K=\operatorname{round}\left(\log _{2} S\right)$, round - функция целочисленного округления, а $a \operatorname{div} b$ - операция целочисленного деления $a$ на $b$.

Для каждого $j$-го признака, $j=1,2, \ldots, N$, оценить индивидуальную информативность $I_{j}$ на основе одной из мер, рассмотренных в [12] (например, можно использовать модуль коэффициента парной корреляции, коэффициент корреляции знаков, коэффициент корреляции Фехнера и др.), после чего определить ранг каждого $j$-го признака $r_{j}$ $\in[N, N-1, \ldots, 1]$ в порядке убывания значений информативности признаков.

Этап 2. Отображение обучающей выборки на обобщенную ось. На основе заданного преобразования определить координату каждого экземпляра на обобщенной оси. 


\section{ТЕОРІЯ І ПРАКТИКА АВТОМАТИЗАЩЇ БІЗНЕС-ПРОЦЕСІВ}

Для задач большой размерности целесообразно обеспечить использование таких преобразований, которые позволяли бы выполнять отображение отдельных экземпляров без загрузки всей исходной выборки, а также в процессе преобразования учитывали информативность признаков и обеспечивали обобщение данных.

Для обеспечения обобщения близких точек данных предлагается заменить значения признаков на номера интервалов значений, предварительно дискретизировав признаки. Для разбиения признаков на интервалы номер интервала (терма), в который попал $s$-й экземпляр по $j$-му признаку, предлагается определять по формуле:

$$
\hat{x}_{j}^{s}=\left\{\begin{array}{l}
\operatorname{round}\left(1+\frac{x_{j}^{s}-x_{j}^{\min }}{\theta_{j}}\right), \theta_{j}>0 ; \theta_{j}=\frac{x_{j}^{\max }-x_{j}^{\min }}{k_{j}}, k_{j}=\left\{\begin{array}{l}
K, K>\operatorname{round}(\ln S), K<\sqrt[N]{S} ; \\
\max \{2, \operatorname{round}(\sqrt[N]{S})\}, K>\operatorname{round}(\ln S), K>\sqrt[N]{S} \\
\max \{2, \operatorname{round}(\ln S)\}, K<\operatorname{round}(\ln S)<\sqrt[N]{S} \\
K, \operatorname{round}(\ln S) \leq K, K<\sqrt[N]{S} \\
\max \{2, \operatorname{round}(\sqrt[N]{S})\}, \operatorname{round}(\ln S) \leq K, K \geq \sqrt[N]{S}
\end{array}\right.
\end{array}\right.
$$

Для отображения экземпляров из исходного пространства признаков на одномерную обобщённую ось предлагается использовать следующие преобразования.

Преобразование 1 (см. рис. 1а). Для каждого номера интервала $j$-го признака получим его бинарное представление (бинарные числа, дополненные нулями слева до $c_{j}$ - числа цифр в $k_{j}$ ). Установим: координату $s$-го экземпляра по обобщённой оси $I^{s}=0$, номер разряда координаты по обобщённой оси $p=1$. Двигаясь по номерам признаков $j$ в порядке убывания их рангов $r_{j}$ и по группе цифр в номере интервала $c=1,2, \ldots, c_{j}$ выполнять в цикле: если $p \leq d$, где $d$ - число разрядов в бинарной разрядной сетке ЭВМ, то записать в $p$-й разряд (при нумерации слева) бинарного представления обобщённого признака $I^{s} c$-й разряд (при нумерации слева) номера интервала, в который попал $s$-й экземпляр по $j$-му признаку $\hat{x}_{j}^{s}$, и принять: $p=p+1$. В результате мы получим координату экземпляра по обобщенной оси с неявным ранжированием и отбором признаков.

Преобразование 2 (см. рис. 1б). Представляет собой альтернативный преобразованию 1 формат построения обобщённого признака. Если суммарное число бит для представления номеров интервалов всех признаков $c_{j} k_{j} N$ не превышает числа разрядов в бинарной разрядной сетке $d$, при равном числе $c_{j}$ для всех признаков: для каждого номера интервала $j$-го признака получим его бинарное представление (бинарные числа, дополненные нулями слева до $c_{j}$ - числа цифр в $k_{j}$ ), установим: координату $s$-го экземпляра по обобщённой оси $I^{s}=0$, номер разряда координаты по обобщённой оси $p=1$; двигаясь в цикле по группе цифр в номере интервала $c=1,2, \ldots, c_{j}$ и по номерам признаков $j$ в порядке убывания их рангов $r_{j}$ : записать в $p$-й разряд (при нумерации слева) бинарного представления обобщённого признака $I^{s} c$-й разряд (при нумерации слева) номера интервала, в который попал $s$-й экземпляр по $j$-му признаку, и принять: $p=p+1$. В результате мы получим координату по обобщенной оси с неявным ранжированием признаков.

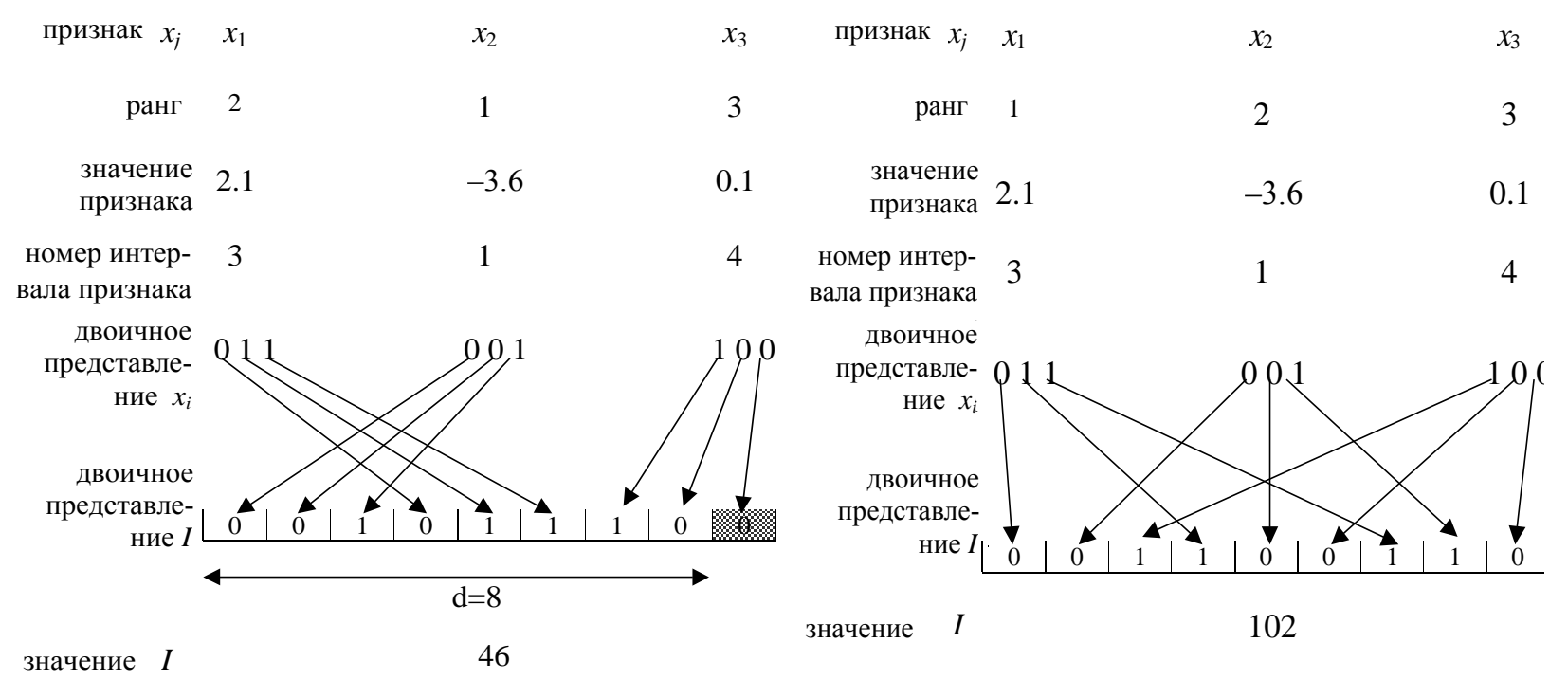


Этап 3. Одномерный кластер-анализ. Упорядочить экземпляры по возрастанию координаты на обобщённой оси. Просматривая слева направо экземпляры на обобщённой оси $I$, определить левые и правые граничные значения одномерных кластеров (интервалов), считая кластером группу экземпляров, относящихся к одному и тому же классу, расположенных рядом на обобщенной оси и непересекающуюся с экземплярами других кластеров. Выделить экземпляры каждого $q$-го кластера.

Этап 4. Многомерный кластер-анализ. Для экземпляров каждого кластера обобщённой оси $I$ определить координаты эталона (центра) кластера в исходной системе признаков $x$ :

$$
C_{j}^{q}=\frac{1}{S^{q}} \sum_{s=1}^{S}\left\{x_{j}^{s} \mid x^{s} \in q\right\}, q=1,2, \ldots, Q,
$$

где $S^{q}$ - число экземпляров, попавших в $q$-й кластер, $Q$ - общее число кластеров. После чего определить значение коэффициента, регулирующего величину кластера:

$$
\beta_{q}=\frac{S^{q}}{\max _{\substack{q=1,2, \ldots, Q ; \\ p=q+1, \ldots, Q}}\left\{\sqrt{\sum_{j=1}^{N}\left(C_{j}^{q}-C_{j}^{p}\right)^{2}}\right\}} .
$$

Этап 5. Синтез структуры РБНС. Задать на первом (скрытом) слое РБНС $Q$ нейронов, использующих в качестве весовых (постсинаптических функций) функции расстояния

$$
\varphi^{(1, i)}\left(x^{(1, i)}, w^{(1, i)}\right)=w_{0}^{(1, i)} \sqrt{\sum_{j=1}^{N}\left(x_{j}^{(1, i)}-w_{j}^{(1, i)}\right)^{2}},
$$

где $w_{0}^{(1, i)}$ - параметр, регулирующий ширину радиально-базисной функции $i$-го нейрона первого слоя, а в качестве активационных функций - функции Гаусса: $\psi^{(1, i)}\left(\varphi^{(1, i)}\right)=\exp \left(-\varphi^{(1, i)}\right)$.

На втором (выходном) слое РБНС задать число нейронов, равное размерности выходного вектора. Определить в качестве весовых функций нейронов второго слоя сети взвешенные суммы

$$
\varphi^{(2, i)}\left(x^{(2, i)}, w^{(2, i)}\right)=\sum_{j=1}^{N_{M}} w_{j}^{(2, i)} x_{j}^{(2, i)}+w_{0}^{(2, i)},
$$

где $w_{0}^{(1, i)}-$ порог, а в качестве активационных функций - линейные функции: $\psi^{(2, i)}\left(\varphi^{(2, i)}\right)=\varphi^{(2, i)}$.

Этап 6. Настройка параметров РБНС. Занести в $j$-й вес $q$-го нейрона первого слоя РБНС значение $j$-й координаты центра $q$-го кластера: $w_{j}^{(1, q)}=C_{j}^{q}, j=1,2, \ldots, N, q=1,2, \ldots, K$. Занести в нулевые веса нейронов первого слоя параметры, регулирующие ширину кластеров: $w_{0}^{(1, q)}=\beta_{q}, q=1,2, \ldots, K$. Определить значения весов нейронов второго слоя РБНС путем решения системы линейных алгебраических уравнений вида $y_{i}=\psi^{(1, i)}\left(\varphi^{(1, i)}(x)\right) w^{(2)}$, где $y_{i}=\left(\left\{y_{i}^{s}\right\}\right)^{T}$ - вектор выходных значений, $w^{(2, i)}-$ вектор весов $i$-го выходного нейрона [9], либо на основе обратного распространения ошибки $[8,10,11]$.

Этап 7. Оптимизация (контрастирование) РБНС. Выполнить распознавание обучающей выборки с помощью построенной РБНС и определить ошибку сети $E$. До тех пор, пока ошибка является приемлемой $(E \leq \varepsilon)$ выполнять последовательно в цикле контрастирование весов второго слоя сети: среди весов второго слоя определить вес с наименьшим абсолютным значением, неравным нулю, положить его равным нулю, выполнить распознавание обучающей выборки, оценить ошибку распознавания $E$. В результате выполнения этих действий часть весов будет обнулена, т.е. можно считать, что будут удалены соответствующие связи, что позволит повысить интерпретабельность полученной модели, а также её уровень обобщения по отношению к исходным данным и дообучение.

Этап 8. Дообучение РБНС. Если имеется обученная сеть, для которой отсутствует исходная обучающая выборка, но имеется ряд новых наблюдений, то возможно реализовать дообучение такой сети. В этом случае необходимо исключить из нового набора наблюдений те, которые не несут новой информации, а оставшиеся занести в сеть. Для этого необходимо выполнить распознавание новых наблюдений относительно обученной сети и определить ошибку распознавания для каждого экземпляра. Все безошибочно распознанные экземпляры можно считать изученными сетью и исключить из набора новых наблюдений.

Для каждого из оставшихся ошибочно распознанных экземпляров $x^{s}$ необходимо сформировать отдельный кластер $q$, добавив на первый слой сети дополнительный нейрон, занести значения признаков экземпляра в 


\section{ТЕОРІЯ I ПРАКТИКА АВТОМАТИЗАЦЇ БІЗНЕС-ПРОЦЕСІВ}

координаты центра соответствующего кластера $C_{j}^{q}=x_{j}^{s}, j=1,2, \ldots, N$, установить значение параметра, регулирующего ширину границ кластера:

$$
\beta_{q}=\frac{1}{\max _{\substack{q=1,2, \ldots, Q ; \\ p=q+1, \ldots, Q}}\left\{\sqrt{\sum_{j=1}^{N}\left(C_{j}^{q}-C_{j}^{p}\right)^{2}}\right\}} .
$$

установить веса связей нейронов второго слоя от нового кластера до соответствующих нейронов второго слоя подобно этапу 6.

Предложенный метод позволяет автоматизировать процесс синтеза РБНС, сокращая зависимость от пользователя, а также учитывает информативность признаков при формировании кластеров.

\section{Эксперименты и результаты}

Для проверки работоспособности и практической применимости предложенного метода он был программно реализован. Разработанная программа использовалась для построения диагностических моделей в задачах технического и медицинского диагностирования [13]: диагностирование лопаток газотурбинных авиадвигателей по спектрам свободных затухающих колебаний после ударного возбуждения (DL), прогнозирование прочности лопаток газотурбинных авиадвигателей после упрочняющей обработки (UL), диагностирование хронического обструктивного бронхита (DB). В таблице приведены характеристики решавшихся задач, а также экспериментально полученные результаты.

Таблица - Характеристики задач и методов синтеза РБНС

\begin{tabular}{|c|c|c|c|c|c|c|c|c|c|c|c|c|}
\hline \multirow{3}{*}{ Задача } & \multicolumn{3}{|c|}{$\begin{array}{l}\text { Характеристики } \\
\text { задачи }\end{array}$} & \multicolumn{9}{|c|}{ Методы синтеза РБНС } \\
\hline & \multirow[t]{2}{*}{$N$} & \multirow[t]{2}{*}{$S$} & \multirow[t]{2}{*}{$K$} & \multirow[t]{2}{*}{$\begin{array}{l}\text { Прямое } \\
\text { выборки } \\
\text { обратное } \\
\text { ошибки } \\
t_{\text {об, с }}\end{array}$} & \multicolumn{2}{|c|}{ 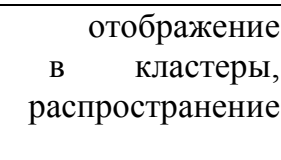 } & \multicolumn{3}{|c|}{\begin{tabular}{|lr} 
Четкий кластер & анализ с \\
сокращением & числа \\
кластеров, & обратное \\
распространие ошибки
\end{tabular}} & \multicolumn{3}{|c|}{ Предложенный метод } \\
\hline & & & & & $E$ & $F_{g}$ & $t_{\text {об., }, \mathrm{c}}$ & $E$ & $F_{g}$ & $t_{\text {об., }} \mathrm{c}$ & $E$ & $F_{g}$ \\
\hline $\mathrm{DL}$ & 100 & 32 & 2 & 1.3 & $0,98 \cdot 10^{-6}$ & 3.48 & 4.9 & $0,94 \cdot 10^{-6}$ & 4.05 & 1.6 & $0,97 \cdot 10^{-6}$ & 4.64 \\
\hline UL & 16 & 57 & 2 & 0.8 & $0.96 \cdot 10^{-6}$ & 3.37 & 3.1 & $0.89 \cdot 10^{-6}$ & 3.62 & 1.2 & $0.92 \cdot 10^{-6}$ & 4.15 \\
\hline DB & 28 & 205 & 2 & 1.6 & $0,91 \cdot 10^{-6}$ & 4.07 & 6.2 & $0,93 \cdot 10^{-6}$ & 5.15 & 2.3 & $0,89 \cdot 10^{-6}$ & 6.89 \\
\hline
\end{tabular}

Для оценивания обобщающих свойств полученных моделей в таблице использован показатель:

$$
F_{g}=\frac{N S}{N_{w}-N_{w=0}},
$$

где $N_{w}$ - общее число весов РБНС, а $N_{w=0}$ - число весов РБНС, равных нулю. Чем больше будет значение данного показателя, тем выше уровень обобщения сетью данных обучающей выборки, тем меньше и компактнее модель, тем она более интерпретабельная.

Как видно из табл. 1, предложенный метод характеризуется меньшими затратами времени на построение РБНС по сравнению с использованием четкого переборного кластерного анализа и большими затратами времени по сравнению с отображением выборки в кластеры. При этом предложенный метод обеспечивает приемлемую точность и существенно более высокий уровень обобщения по сравнению с известными методами.

\section{Выводы}

В работе решена актуальная проблема синтеза РБНС на основе набора прецедентов для автоматизации принятия решений в диагностике.

Научная новизна работы состоит в том, что впервые предложен метод синтеза РБНС, который использует отображение выборки из многомерного пространства признаков на одномерную обобщенную ось для выделения центров и границ кластеров и, в отличие от известных методов, не требует задания пользователем числа кластеров, не имеет неопределенности выбора числа нейронов в первом слое и выбора начальных значений весов сети, стремится минимизировать размер сети, характеризуется приемлемым временем обучения, учитывает информативность признаков при формировании разбиения на кластеры, благодаря использованию процедуры оптимизации сети позволяет получать безызбыточные контрастные интерпретабельные модели, а также обеспечивает возможность дообучения ранее построенных моделей. Практическая значимость результатов работы 
заключается в том, что разработано программное обеспечение, реализующее предложенный метод, а также проведены эксперименты, подтвердившие работоспособность разработанного математического обеспечения и позволяющие рекомендовать его для использования на практике при решении задач построения диагностических моделей по прецедентам для автоматизации принятия решений в технической и биомедицинской диагностики. Перспективы дальнейших исследований состоят в исследовании предложенного математического обеспечения на более широком наборе практических задач диагностики и распознавания образов, а также в изучении вопроса автоматического выбора наилучшего преобразования на обобщенную ось из набора заданных преобразований при использовании предложенного метода. Работа выполнена в рамках в рамках госбюджетных научноисследовательских тем Запорожского национального технического университета "Интеллектуальные информационные технологии диагностирования и автоматической классификации” и “Интеллектуальные методы диагностирования систем управления удаленными техническими объектами”. при поддержке международного проекта "Centers of Excellence for young RESearchers” программы "Темпус" Европейской Комиссии (№ 544137TEMPUS-1-2013-1-SK-TEMPUS-JPHES).

\section{Литература}

1. Intelligent fault diagnosis and prognosis for engineering systems / [G. Vachtsevanos, F. Lewis, M. Roemer et al.]. - New Jersey: John Wiley \& Sons, 2006. - 434 p.

2. Price C. Computer based diagnostic systems / C. Price. - London: Springer, 1999. - 136 p.

3. Дли М. И. Нечёткая логика и искусственные нейронные сети / М. И. Дли. - М.: Физматлит, 2003. - 225 с.

4. Рутковская Д. Нейронные сети, генетические алгоритмы и нечёткие системы / Д. Рутковская, М. Пилиньский, Л. Рутковский; пер.польск. И. Д. Рудинского.-М.:Горячая линия-Телеком, 2004. - 452 с.

5. Rao V. B. C++ neural networks and fuzzy logic / V. B. Rao. - Foster City: M\&T Books, 1995. - 549 p.

6. Bishop C. Neural networks for pattern recognition / C. Bishop. - New York: Oxford University Press, 1995 . - 482

7. Круглов В. В. Искуственные нейронные сети. Теория и практика / В. В. Круглов, В. В. Борисов. - М.: Горячая линия - Телеком, 2001. - 382 с.

8. Осовский С. Нейронные сети для обработки информации / - М.: Финансы и статистика, 2004. - 344 с.

9. Руденко О. Г. Штучні нейронні мережі / О. Г. Руденко, С. В. Бодянський. - Харків: СМІТ, $2006 .-404$ с.

10. Хайкин С. Нейронные сети: полный курс / С. Хайкин. - СПб: Вильямс, 2005. - 1104 с.

11. Субботін С. О. Нейронні мережі : навч. посіб. / С. О. Субботін, А. О. Олійник; за ред. С. О. Субботіна. Запоріжжя : ЗНТУ, 2014. - 132 с.

12. Олійник А. О. Інтелектуальний аналіз даних : навчальний посібник / А. О. Олійник, С. О. Субботін, О. О. Олійник. - Запоріжжя : ЗНТУ, 2012. - 278 с.

13. Интеллектуальные информационные технологии проектирования автоматизированных систем диагностирования и распознавания образов : монография / [С. А. Субботин, Ан. А. Олейник, Е. А. Гофман и др.] ; под ред. С. А. Субботина. - Харьков : ООО «Компания Смит», 2012. - 317 с.

\section{References}

1. G. Vachtsevanos, F. Lewis, M. Roemer et al., Intelligent fault diagnosis and prognosis for engineering systems. New Jersey: John Wiley \& Sons, 2006.

2. C. Price, Computer based diagnostic systems. London: Springer, 1999.

3. M. I. Dli, Nechyotkaya logika i iskusstvennye nejronnye seti. Moskva: Fizmatlit, 2003.

4. D. Rutkovskaya, M. Pilin'skij, L. Rutkovskij, Nejronnye seti, geneticheskie algoritmy i nechyotkie sistemy. Moskva: Goryachaya liniya - Telekom, 2004.

5. V. B. Rao, C++ neural networks and fuzzy logic. Foster City: M\&T Books, 1995.

6. C. Bishop, Neural networks for pattern recognition. New York: Oxford University Press, 1995.

7. V. V. Kruglov, V. V. Borisov, Iskustvennye nejronnye seti. Teoriya i praktika. Moskva:Goryachaya liniya Telekom, 2001

8. S. Osovskij, Nejronnye seti dlya obrabotki informacii. Moskva: Finansy i statistika, 2004.

9. O. G. Rudenko, Je. V. Bodjans'kyj, Shtuchni nejronni merezhi. Harkiv: SMIT, 2006.

10. S. Xajkin, Nejronnye seti: polnyj kurs. Sankt-Peterburg: Vil'yams, 2005.

11. S. O. Subbotin, A. O. Olijnyk, Nejronni merezhi : navch. posib. Zaporizhzhja : ZNTU, 2014.

12. A. O. Olijnyk, S. O. Subbotin, O. O. Olijnyk, Intelektual'nyj analiz danyh : navchal'nyj posibnyk. : ZNTU, 2012.

13. S. A. Subbotin, An. A. Olejnik, E. A. Gofman i dr., Intellektual'nye informacionnye texnologii proektirovaniya avtomatizirovannyx sistem diagnostirovaniya i raspoznavaniya obrazov: monografiya. Xar'kov : OOO «Kompaniya Smit», 2012. 